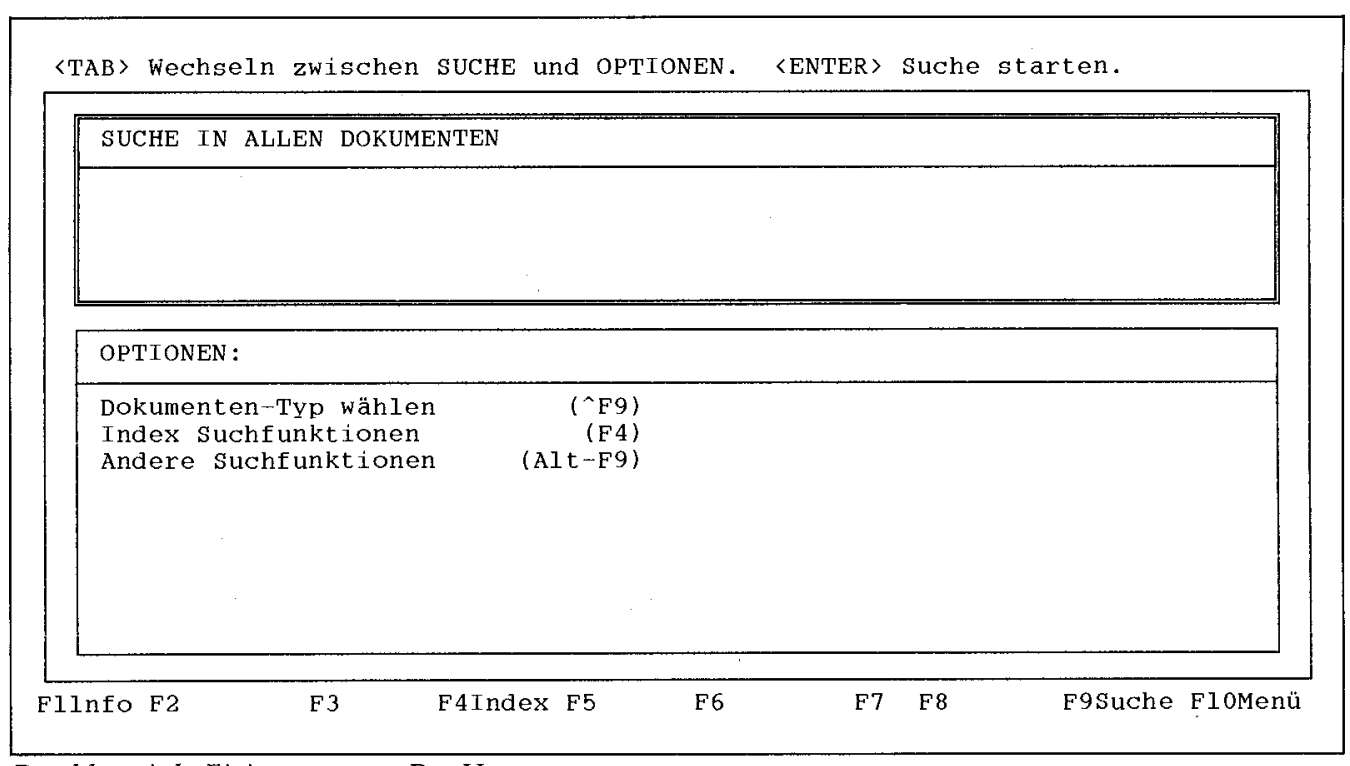

Der elektronische Einigungsvertrag: Das Hauptmenï

\section{Mit dem Einigungsvertrag arbeiten}

Einige-praktische Anmerkungen anläßlich des Erscheinens der jur-pc CD-ROM Edition

\section{Maximilian Herberger}

Das „Zeitfenster" für die Vereinigung der (ehemals) beiden deutschen Staaten war eng. Wie eng, wird langsam immer deutlicher. Insofern ziehen schon jetzt alle die Beiträge das historische Interesse aufi sich, die für das Einhalten des knappen Fahrplanes unentbehrlich waren. Nicht an letzter Stelle rangiert dabei die Leistung der Ministerialbürokratie. Die Einschätzung, daß ohne deren Sachgebietsroutine ein Vertragswerk wie der Einigungsvertrag in dem vorgegebenen Zeitraum nicht zu erstellen gewesen wäre, ist ebenso richtig, wie sie noch der Aufnahme durch das öffentliche Bewußtsein harrt. Gerade weil die Bürokratie eine dienende Rolle spielt, stehen die Chancen, daß ihre besondere Anstrengung und Leistung die gebührende Anerkennung erfährt, nicht besonders gut. Insofern sei dieser Vorspann all denen gewidmet, die einerseits pflichtgemäß und andererseits doch überobligationsmäßig den Text mitgestaltet haben, dessen arbeitspraktische Bewältigung im folgenden besprochen werden soll: Den Einigungsvertrag.

Mit dem Stichwort „arbeitspraktisch“ ist bereits das angedeutet, was gewissermaßen die Kehrseite der Medaille des Arbeitsrhythmus bei der Erstellung des Vertragstextes darstellt: Mit dem Text des Einigungsvertrages kann so, wie er im Bundesgesetzblatt veröffentlicht worden ist, nicht sinnvoll gearbeitet werden. Dabei liegt das Problem weniger in dem Vertragstext selbst: Dieser ist mit 45 Artikeln so kurz, daß man ihn aufi der Suche nach einer Antwort nahezu jedesmal kursorisch überfliegen kann, ohne allzuviel Zeit zu verlieren. (Obwohl auch das manchen ungeduldigen Leser schon ärgerlich gestimmt hat.) Das wahre Problem sind die Anlagen. Einige Hinweise mögen das veranschaulichen.

\section{Kein Inhaltsverzeichnis - und die Folgen}

Der Text im Bundesgesetzblatt hat - anders als sonst bei Gesetzen üblich - kein Inhaltsverzeichnis. Falls man also etwa irgendwo eine Referenz aufi „Anlage I, Kapitel III, Sachgebiet B, Abschnitt II" findet, ist ein gezielter Zugriffi nicht möglich. Ein zeitraubendes und ärgerliches Blättern wird unausweichlich. Manche Leser des Bundesgesetzblattes sind deswegen dazu übergegangen, ihr Exemplar des Einigungsvertrages mit über den Buchblock hinausragenden „Blätterhilfen“ zu versehen. Diese erlauben dann ein direktes Aufblättern der gesuchten Systemstelle. Indessen ist der Zeitaufwand für das Anbringen dieser „Blätterhilfen“ an den Seiten recht hoch und keinesfalls erfreulich.

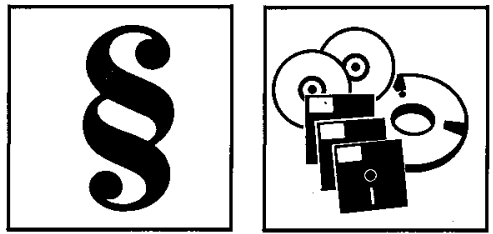

Noch nicht'genügendigezeürdigt:

'Die Leistung der

Ministerialbürokratie

Mit dem Text im BGBl. kann nicht sinnvoll'gearbeitet werden. 


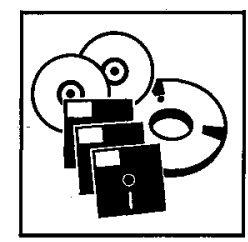

Man sollte nach der Seite im $B G B l$ zitieren.

Viel. Blätterei auch bei den internen Querverweisen

Man könnte nun einwenden, daß man dem geschilderten Problem entgehen könnte, wenn man den Einigungsvertrag nach der Seite im Bundesgesetzblatt zitieren würde und nicht nach einer schwer aufzufindenden Systemstelle. Das wäre in der Tat eine sinnvolle Konvention.

(Die jur-pc Ausgabe des Einigungsvertrages enthält deswegen die Seiten des Bundesgesetzblattes als Randziffern und die CD-ROM unterstützt eine entsprechende Zitiergewohnheit dadurch, daß sie stets die Seite des Bundesgesetzblattes mit anzeigt und auch einen direkten Zugriffi zu einer Bundesgesetzblatt-Seite erlaubt.)

Indessen zeichnet sich eine Konvention, nach dem Bundesgesetzblatt zu zitieren, auch nicht annähernd $a b$. Drei Beispiele seien stellvertretend für viele angeführt:

- Wer den (sehr lesenswerten) Ausführungen von Battis zum Thema „Rechtsstaatliche Verfahren zur Erneuerung der Hochschulen“" (FAZ, 27.12.90, S. 6) folgen und diese nachvollziehen will, wird sich unweigerlich aufi die Suche nach „Anlage 1 zum Einigungsvertrag im Kapitel XIX Sachgebiet A Abschnitt III in der Ziffer 1" machen müssen. Hat der geneigte Leser dabei nur das Bundesgesetzblatt zur Hand - siehe oben.

- In der „Deutsch-Deutschen Rechtszeitschrift“ beschäftigt sich Ronald Brachmann mit dem Thema „Die Gerichtsverfassung im Übergang -Zur Regelung auf dem Gebiet der ehemaligen DDR nach dem Einigungsvertrag“" (1990, S. 298 - 305). In Fußnote 10 heißt es: „Diese und alle anderen nicht näher bezeichneten Quellenangaben beziehen sich auf Anl. I zum Einigungsvertrag, Kap. III, Sachgeb. A Abschn. III Nr. 1.“

- Gleichfalls in der DtZ behandeln Thomas Pfeiffer und Daniela Birkenfeld-Pfeiffer das „Arbeitsrecht nach dem Einigungsvertrag“ (1990, S. 325 - 330). Im vorliegenden Kontext sei dort beispielsweise verwiesen auf Fußnote 28 („Anl. II Kap. X Sachgeb. $\mathrm{H}$ Abschn. III Nr.1") und die folgenden Fußnoten.

Selbst wenn sich übrigens die eben empfohlene Zitierkonvention nach den Seitenzahlen des Bundesgesetzblattes durchsetzen sollte, des Blätterns wäre trotzdem kein Ende. Der Einigungsvertrag enthält nämlich interne Querverweisungen, die dem gerade besprochenen Schema folgen. Betrachten wir einige Beispiele:

- „für die Weiterverwendung von Personen im öffentlichen Dienst (Anlage I Kapitel XIX Sachgebiet A Abschnitt III Nr. 1)“ (BGBl. II 1990, 913).

- „Die Bestimmungen der $\S \S 4$ bis 6 über die entsprechende Anwendung des Bundesversorgungsgesetzes und der zu seiner Durchführung erlassenen Vorschriften gelten in dem in Artikel 3 des Vertrages genannten Gebiet mit den in Anlage I Kapitel VIII Sachgebiet K Abschnitt III aufgeführten Maßgaben“ (BGBl. II 1990, 920).

- „Konkursverfahren im Sinne dieser Vorschrift kann auch ein Verfahren nach der Gesamtvollstreckungsordnung (Anlage II Kapitel III Sachgebiet A Abschnitt II Nr. 1) sein" (BGBl. II 1990, 935).

Es mag damit (insgesamt gibt es 44 Querverweisungen dieses Typs im Einigungsvertrag) sein Bewenden haben. Die Auswahl legt einen Eindruck nahe, den schon mancher Leser dieses Textes hatte: Man würde sich hier ein „festes Verdrahten“ wünschen, das wiederum durch Angabe der entsprechenden Seitenzahl im Bundesgesetzblatt möglich wäre - zu Blättern hätte man dann allemal noch genug. Noch schöner wäre allerdings eine „papierfreie“ Art der elektronischen Aufbereitung, von der noch zu sprechen sein wird.

\section{Kein Index - und die Folgen}

Der Text des Einigungsvertrages im Bundesgesetzblatt weist keinen Index auf einen Stichwortindex noch einen Index der in Bezug genommenen Normen. Das erscheint zunächst nicht als unüblich: Gesetze verfügen üblicherweise über kein Register. Sie brauchen es unter normalen Umständen auch nicht. Das hat seinen Grund darin, daß Gesetze typischerweise systematisch in einer Art gegliedert sind, die den Zugriff auf relevante Regelungen ohne Unterstützung durch ein Register erlaubt. (Das führt auch zu der häufig anzutreffenden pädagogischen Verurteilung der Registernutzung, die mancher Leser noch aus seinen Studienzeiten kennen wird.) Beim Einigungsvertrag ist aber die regelmäßig angenommene systematische Ideallage in den Anlagen (vorsichtig ausgedrückt) nur annähernd realisiert. 
Bilden wir zur Veranschaulichung ein Beispiel: Vor der zuständigen Behörde erscheint ein Falkner und fragt, ob seine vor dem Wirksamwerden des Beitritts abgelegte Jagdprüfung für Falkner weiter Gültigkeit hat. Die (bejahende) Antwort findet sich in Anlage I Kapitel VII (Geschäftsbereich des Bundesministers für Ernährung, Landwirtschaft und Forsten) im Sachgebiet F (Forstwirtschaft, Jagdwesen und Fischerei) und dort im Abschnitt II = BGBl. II 1990, 1017. (Übrigens zugleich ein schönes Beispiel dafür, an welche Einzelheiten man bei der Ausarbeitung des Vertrages gedacht hat.)

Der Weg vom Problem zur Lösung ist also nicht einfach. Nun könnte man einwenden, daß das Beispiel des Falkners nicht gerade breite Bevölkerungskreise betrifft. Indessen würde diese Beobachtung dem Problem seine Bedeutung nicht nehmen: Auch wenn es um nur eine Person ginge, wäre die richtige Behandlung geboten. Gewichtiger ist vielleicht der Einwand, daß ein mit der Zuständigkeitsordnung der Ministerien vertrauter Leser die Stelle (ein gutes Inhaltsverzeichnis vorausgesetzt, das dem Bundesgesetzblatt fehlt, aber in einzelnen Textausgaben in unterschiedlicher Tiefe vorhanden ist) die relevante Textstelle recht direkt finden könnte. Das ist aber nicht immer so. Wer etwa würde sich auf Anhieb zutrauen den Zuständigkeitsbereich zu benennen, innerhalb dessen geregelt werden müßte, ob (und $a b$ wann) das Fünfte Vermögensbildungsgesetz in der früheren DDR Anwendung findet? (Man lese für die Antwort BGBl. II 1990, 1070).

Und um der Problematik noch eine schärfere Dimension zu geben, soll folgender Fall betrachtet werden:

Angenommen, es sei unter der Geltung des früheren DDR-Rechts ein Vertrag geschlossen worden. Es fragt sich nun, ob für diesen Vertrag das DDR-Recht im Zeitpunkt des Vertragsschlusses weitergilt. Die grundsätzliche Regel für die Antwort (möglichen Ausnahmen gilt es je nach Vertragsinhalt und Vertragstyp noch ergänzend nachzugehen) findet sich im Einigungsvertrag in folgender Form: „Für ein Schuldverhältnis, das vor dem Wirksamwerden des Beitritts entstanden ist, bleibt das bisherige für das in Artikel 3 des Einigungsvertrages genannte Gebiet geltende Recht maßgebend“ (BGBl. II 1990, 943).

Summa summarum: Ein Index wäre hilfreich - übrigens eine Erkenntnis, die sich durchzusetzen beginnt: „Ein ausführlicheres ... Glossar ... wäre dringend zu wünschen“ (Fromme, FAZ, 30.11.1990, S. 5). Die jur-pc-Ausgabe des Einigungsvertrages wies deswegen von Anfang an einen solchen Index sowohl in der Form des Textwort-Indexes als auch der Form eines Verzeichnisses der betroffenen Gesetze aufı Indessen stößt die Präsentation eines Indexes in gedruckter Form schnell an materialmäßige Grenzen. Wiederum wäre eine elektronische Unterstützung wünschenswert - idealiter als Völltextsuche, da dann der denkbar größte Textwortindex zur Verfügung steht. Eine erste Stufe der elektronischen Präsentation des Registers stellte die Diskettenfassung dar, die zumindest all denen das zeitraubende Hin- und Herblättern im Buch reduziert, die den PC zum selbstverständlichen Arbeitsinstrument aufı dem Schreibtisch zählen.

\section{Der Einigungsvertrag elektronisch - und das juristische Bewußtsein}

Gerade weil der Einigungsvertrag sich so präsentiert, wie er sich präsentiert, sind Orientierungshilfen „zu dem verwinkelten Gebäude des Einigungsvertrages mit seinen geheimen Gängen und blinden Stollen“ (Fromme, FAZ, 30.11.1990, S. 5) unentbehrlich. $\mathrm{Daß}$ man in der Papierumgebung dabei schnell an Grenzen stößt, ist deutlich geworden: Ein Index mit allen im Text vorkommenden Worten etwa ist im Druck undenkbar. Insofern bietet gerade dieses Regelungswerk die Chance, Juristen mit elektronischen Erschließungsformen vertraut $\mathrm{zu}$ machen, die sie ansonsten aufgrund gut vorhandener anderer Strukturierungshilfen abzulehnen geneigt sind. Dies war zwar bei der Edition des Einigungsvertrages auf CD-ROM nur ein Nebengedanke. Im Vordergrund stand die Idee, diesen zentralen Text in der bestmöglichen Form zugänglich zu machen. Die ersten Erfahrungen zeigen indessen, daß die beschriebene psychologische Nebenwirkung durchaus häufig eintritt. Ursache dafür ist schlicht und einfach, daß sehr viele papiergestützte Suchen im Einigungsvertrag fehlschlagen und sich dann die Suche aufı der CD-ROM als letzte Möglichkeit anbietet. Übrigens verbindet sich gerade mit dem Phänomen des Fehlschlagens der Suche ein bedeutsamer Unterschied der gewohnten Papiermedien und der elektronischen Medien. Wer etwa der Frage nachgeht, ob die gesetzliche Regelung zur Organtransplantation aus dem Recht der früheren DDR

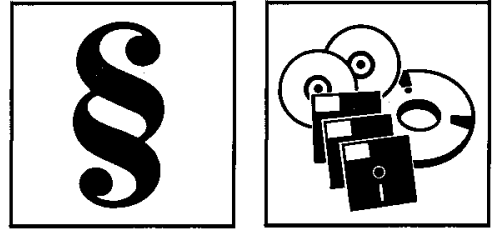

Beispiel.1:

Gilt die Jagdprüfung, für Falkner weiter?

Beispiel.2:

Findet das Fünfte

Vermägensbildungsgesetz in der früheren DDR Anwendung?

Beispieli3:

Nach welchem Recht leben Schuldverbältnisse zeeiter?

„Ein Glossar wäre dringend.zu wünschen"

„Gebeime Gänge undiblinde Stollen"

Vielerpapiergestützte Suchen schlagen fehl:

Was folgt daraus? 
weitergilt, wird bei Suchversuchen im Text des Einigungsvertrages nach einiger Zeit die Hypothese aufstellen, daß eine Weitergeltung wohl nicht anzunehmen ist. Grund dafür ist, daß eine Anordnung der Weitergeltung nicht gefunden wurde. Allerdings liegt der Sicherheitsgrad dieser Erkenntnis erst dann sehr hoch, wenn alle einschlägigen Textpassagen durchgearbeitet wurden. Tut man das nicht und begnügt sich mit Stichproben, so bewegt man sich auf unsicherem Boden. Quantitativ anders sieht die Lage im elektronischen Umfeld aus. Wegen der dort vorhandenen Volltexterschließung kann man alle Textpassagen binnen kürzester Zeit durchsuchen. Gewißheit ist damit allerdings auch nicht verbunden, das muß der Korrektheit wegen angemerkt werden. Denn die verwendeten Suchworte könnten so gewählt sein, daß sie an der relevanten Textstelle vorbeigehen. Jedoch steigt trotz dieser stets verbleibenden Restunsicherheit die Wahrscheinlichkeit in signifikanter Weise, vom Nicht-Finden aufi das Nicht-Vorhandensein einer Regelung zu schließen.

\section{Die elektronischen Möglichkeiten: Volltextsuche mit Trunkierung und Metrik}

„Stand der Technik“ bei CD-ROM Retrieval-Software ist selbstverständlich die Möglichkeit, nach jedem im Text vorkommenden Wort zu suchen. Die oben erörterte Frage des Falkners nach der Weitergeltung seiner Jagdprüfung wäre so leicht $\mathrm{zu}$ beantworten. (Gesetzt den Fall, das Wort „Falkner" würde nicht gefunden, könnte man auch nahezu

„Standider Technik": Einwort-Suche und LogikVerknüpfung

Suche aus dem Index sicher sein, daß eine spezielle Regelung für Falkner nicht getroffen worden ist. Offen bliebe dann immer noch die Frage, ob eine allgemeinere Regelung für mehrere Berufsgruppen getroffen worden ist, die auch für die in Frage stehende Berufsgruppe einschlägig ist. Das ist schwieriger zu entscheiden.)

Zusätzlich zur Einwort-Suche bieten (soweit ersichtlich) alle Retrieval-Programme die Möglichkeit, mehrere Suchworte durch „und“, „oder" bzw. „nicht“ logisch zu verknüpfen. Die Programme unterscheiden sich in dieser Hinsicht lediglich durch die Leichtigkeit und Übersichtlichkeit der entsprechenden Verknüpfungsoperationen.

Allgemeiner "Stand der Technik“ ist auch, daß der Index aller im Text vorkommenden Worte aufgeblättert werden kann und deshalb die Möglichkeit besteht, aus dem Index heraus Worte in die Suchfrage zu übernehmen (vgl. Abb. 1)

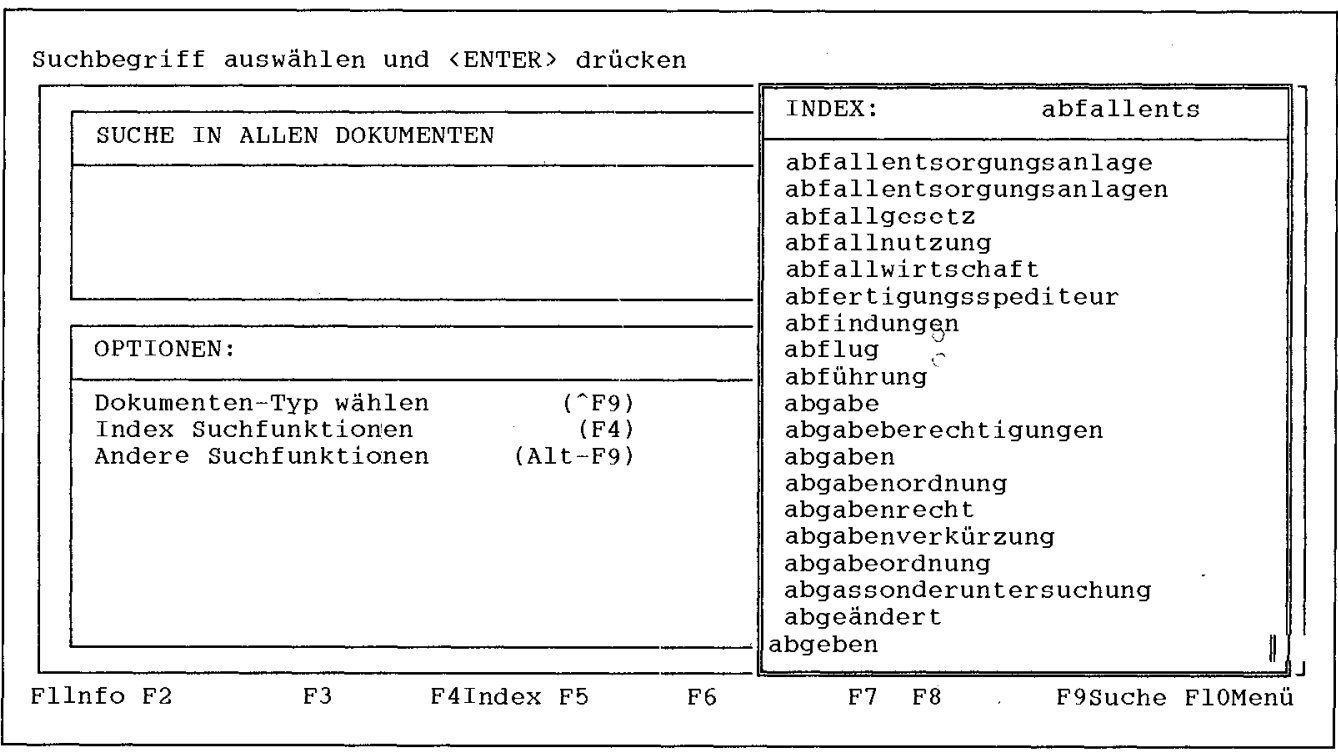

Abb. 1: Der Index.

Der Vorteil dieser Methode gegenüber der „freien“ Eingabe eines Suchwortes liegt nicht nur darin, daß Tapparbeit gespart wird. Hinzu kommt der Vorteil, daß man wegen der alphabetischen Anordnung der Worte das Umfeld eines Suchwortes überblickt und von daher manchmal zu einer Umformulierung der in Aussicht genommenen Suchfrage angeregt wird. Davon wird sogleich bei der Behandlung der Trunkierung näher zu sprechen sein.

Trunkierung In der Frage der Trunkierung beginnt die vorhandene CD-ROM Retrieval-Software sich zu differenzieren. Unter „Trunkierung“ (das nicht sehr schöne Wort ist ein an „truncation" angelehnter Anglizismus) versteht man die Möglichkeit, bei der Suche Stellver- 
treter für beliebige Buchstaben einzugeben. Aufi diese Art und Weise können mit einer Eingabe ganze Wörtfelder abgedeckt werden. Wer etwa „Revision"*s eingibt (der Asterisk * steht für eine beliebige Buchstabenfolge), findet nicht nur „Revisionsgericht", sondern beispielsweise auch „Revisionsinstanz" und „Revisionsverfahren“.

Das eben gewählte Beispiel war ein Fall von Endtrunkierung. Diese Möglichkeit trifft man wohl überall an. Seltener wird zusätzlich Mittel- und Anfangstrunkierung angeboten, wie dies bei der Retrieval-Software CDIS zum Einigungsvertrag der Fall ist. Die Mittel- und Anfangstrunkierung folgt wieder dem eben beschriebenen Prinzip, ist aber -wie leicht ersichtlich ist - aufi andere sprachliche Konstellationen zugeschnitten. In der deutschen Sprache ist vor allem die Anfangstrunkierung deswegen von Bedeutung, weil man damit Vörsilbenvarianten beim selben Grundwort suchökonomisch behandeln kann.

Warum bietet nicht jede Software alle diese wünschenswerten Trunkierungsmöglichkeiten an? Ein Grund dafür liegt darin, daß man für Anfangs- und Endtrunkierung kompliziertere und umfangreichere Indizes anlegen muß. Bei der Endtrunkierung (dem insofern einfacheren Fall), weiß man, an welcher Stelle im Alphabet man mit der Suche $\mathrm{zu}$ beginnen hat. Bei der Anfangstrunkierung ist das zunächst ungewiß und muß erst durch Prüfoperationen bestimmt werden. Bei der Mitteltrunkierung ist zwar der Einstiegspunkt bekannt, danach aber können die von der trunkierten Formulierung erfaßten Suchworte verstreut im Alphabet stehen.

Unter Metrik versteht man im vorliegenden Kontext die Möglichkeit, Suchworte in einem bestimmten Abstand voneinander zu suchen. Dabei sind prinzipiell unterschiedliche Abstandsmaße möglich. In der jur-pc CD-ROM zum Einigungsvertrag kann der Abstand in Worten, Sätzen oder Absätzen bestimmt werden.

Der (statistisch gesehen) richtige Grundgedanke, der hinter dem Suchen mit Metrik steht, besagt, daß nahe beieinander befindliche Worte in einem stärkeren inhaltlichen Zusammenhang stehen als weiter voneinander entfernte Worte. Aufidiese Art und Weise trägt die Metrik dazu bei, das „Störgeräusch“ bei Suchen zu vermindern: Irrelevante Dokumente werden durch die Fokussierung aufi benachbarte Suchworte ausgefiltert.

Metrik ist nicht einmal in allen Online-Datenbanken vorhanden. (juris beispielsweise bietet keine echte Metrik.) Auch zahlreiche CD-ROM gestützte Suchsysteme verfügen nicht über eine Metrik-Möglichkeit. Der Grund hat wieder mit der Komplexität und dem Umfang der für Metrik nötigen Indizes zu tun: Je flexibler die Metrik, desto mehr Positionsangaben müssen mit abgespeichert werden.

Wegen der positiven Auswirkungen der Metrik für die Präzision der Suche sollte aufidas Vorhandensein einer Metrik-Möglichkeit geachtet werden. Man hat es in diesem Punkt mit einem gewichtigen qualitätsunterscheidenden Merkmal $\mathrm{zu}$ tun.

\section{Von großer Bedeutung für die Suche: Die Dokumentenstruktur}

Oft unterschätzt wird die Bedeutung, die der Segmentierung des Textes für die adäquate elektronische Bearbeitung zukommt. Wenn nur eine nicht weiter nach Struktureinheiten differenzierbare Völltextsuche durch einen Gesamttext möglich ist, erreicht die Präzision der Suche tendenziell sehr niedrige Werte. Die Ursache dafür ist einfach zu sehen: Wenn der gesamte Text ein Dokument darstellt, steigt die Wahrscheinlichkeit, daß Suchworte - aufi den ganzen Text gesehen - zusammen vorkommen. Anders ausgedrückt: Man hat (fast) immer Treffer. Nur besagt das ,inhaltlich" nicht besonders viel. Die eben behandelte Metrik bietet eine Möglichkeit, dieser Tendenz entgegenzuwirken. Mit der Metrik allein wird man aber einem Text nicht gerecht, der (längen- und abstandsunabhängig) funktional differenzierte Einheiten enthält. Ein solcher Text ist der Einigungsvertrag mit seinen Anlagen. Die Erschließung für die Suche hat daraufı Rücksicht zu nehmen.

Die Anlagen zum Einigungsvertrag haben jeweils einen eigenen Regelungsgehalt:

- Anlage I enthält besondere Bestimmungen zur Überleitung von Bundesrecht (BGBl. II 1990, 907).

- Anlage II enthält besondere Bestimmungen für fortgeltendes Recht der Deutschen Demokratischen Republik (BGB1. II 1990, 1148).

Innerhalb der Anlagen wird weiter untergliedert nach Geschäftsbereichen der Ministerien, darin nach Sachgebieten und schließlich nach Abschnitten. Die Abschnitte orientieren sich an der Art des gesetzgeberischen Vorgehens.

Funktionale Strukturen im Einigungsvertrag: Anlagen und Abschnitte 


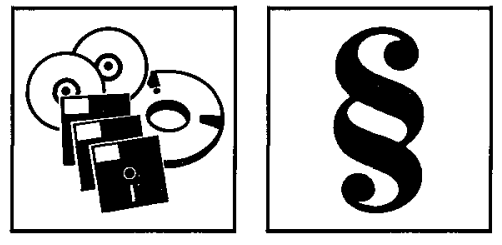

Bedeutung der Abschnitte in Anlage II.

Begrenzung der. Suche auf Anlage und Abscbnitt

Nicht'ganz unberechtigt:

Der Wunsch, das elektronische Medium „aufblättern“'zu können

Ein „dynamisches" Inhaltsverzeichnis

In Anlage I (Besondere Bestimmungen zur Überleitung von Bundesrecht) haben die Abschnitte die folgenden Inhalte (vgl. BGBl. II 1990, 907):

- Abschnitt I: Die hier aufgeführten Rechtsvorschriften sind von dem Inkrafttreten des Bundesrechts in der früheren DDR ausgenommen.

- Abschnitt II: Die hier aufgeführten Rechtsvorschriften werden aufgehoben, geändert oder ergänzt.

- Abschnitt III: Die hier aufgeführten Rechtsvorschriften treten in der früheren DDR mit Maßgaben in Kraft.

In Anlage II (Besondere Bestimmungen für fortgeltendes Recht der Deutschen Demokratischen Republik) haben die Abschnitte die folgenden Inhalte (vgl. BGB1. II 1990, 1148):

- Abschnitt I: Das hier aufgeführte Recht der früheren DDR bleibt in Kraft.

- Abschnitt II: Die hier aufgeführten Rechtsvorschriften der früheren DDR werden aufgehoben, geändert oder ergänzt.

- Abschnitt III: Die hier aufgeführten Rechtsvorschriften der früheren DDR bleiben mit Maßgaben in Kraft.

- Abschnitt IV: Besonderheiten für das Land Berlin.

Folgt man dem Postulat, daß die Suche auf die Strukturen des Textes Rücksicht zu nehmen hat, so ist im Falle des Einigungsvertrages zunächst die Möglichkeit einer Beschränkung der Suche aufi Anlage I bzw. Anlage II vorzusehen. Bei der jur-pc CD-ROM ist diese Eingrenzung über die Option „Wahl des Dokumententyps“ realisiert. Zusätzlich muß es möglich sein, innerhalb der Anlagen die Suche aufiAbschnitte zu begrenzen, da diese in der geschilderten Weise unterschiedliche Bedeutung haben. Diesem Postulat trägt die Funktion „Suche in BGBl.-Abschnitt“ Rechnung.

Handhabt man die bisher beschriebenen Möglichkeiten geschickt, so kann beispielsweise folgendes Suchproblem präzise „aufi den Punkt“ gebracht werden:

Ein bisher nach der „Anordnung über die individuelle Förderung der beruflichen Ausbildung" Geförderter möchte wissen, ob diese Anordnung fortgilt.

Zur Lösung geht man folgendermaßen vor:

- Beschränkung aufi Anlage II (=die Frage betrifft die mögliche Fortgeltung von DDRRecht).

- Beschränkung aufı Abschnitt I (= Anordnung der Fortgeltung von DDR-Recht).

- Suche nach „Förderung“ und „Ausbildung“ (mit Metrik, da sonst auch nicht-einschlägige Treffer mit auftauchen, bei denen von der „Förderung von Steinkohle“ die Rede ist.)

Die Antwort (BGBl. II 1990, 1208fi) wird so schnell und präzise gefunden.

\section{Über die Suche hinaus: Der gezielte Zugrifffaus einer Struktur heraus}

Ein Teil der juristischen Reserven gegenüber elektronischen Informationssystemen beruht darauf, daß diese Systeme ein Umlernen verlangen. Dem Juristen wird bedeutet, daß er zuerst sein gewohntes Suchverhalten aufgeben und dann eine (mehr oder weniger komplizierte) neue Suchsprache lernen muß. Es ist nicht ganz unverständlich, wenn Juristen trotz aller Leistungsfähigkeit der neuen Suchmöglichkeiten sich der Notwendigkeit des Umlernens entziehen.

Gesteht man aber der eben beschriebenen Haltung, die an gewohnten Suchroutinen festhalten will, eine gewisse Berechtigung zu, so muß man zusätzlich zu den neuen, ein Umlernen voraussetzenden Zugriffsmöglichkeiten (aufı die natürlich nicht verzichtet werden kann) auch solche vorsehen, die der gewohnten Arbeitsweise nahestehen. CDIS, die bei der jur-pc CD-ROM eingesetzte Retrieval-Software, bietet etwas Derartiges an. Abstrakt kann die vorgeschaltete Struktur, aus der heraus man.zur Zielinformation gelangt, als hierarchisch organisierte Gliederung beschrieben werden, die an der tiefsten Verzweigungsstufe aufi ein Zieldokument verweist. Konkret realisiert ist diese Struktur im vorliegenden Fall als tiefgestaffeltes Inhaltsverzeichnis (vgl. Abb. 2 aufi der nächsten Seite). Man kann dieses Inhaltsverzeichnis schrittweise „expandieren“, aufi diese Weise eine jeweils speziellere Sicht gewinnen und dann schließlich zu dem dahinterliegenden Text blättern. Dieser Arbeitsgang entspricht dem Arbeiten mit dem Inhaltsverzeichnis und kann (um ihn von der Suche abzugrenzen) als „elektronische Lektüre“ bezeichnet werden. Es ist anzunehmen, daß Juristen durch derartige Angebote leichter dazu motiviert werden können, ein elektronisches Medium zu nutzen, als dies bei exklusiven 


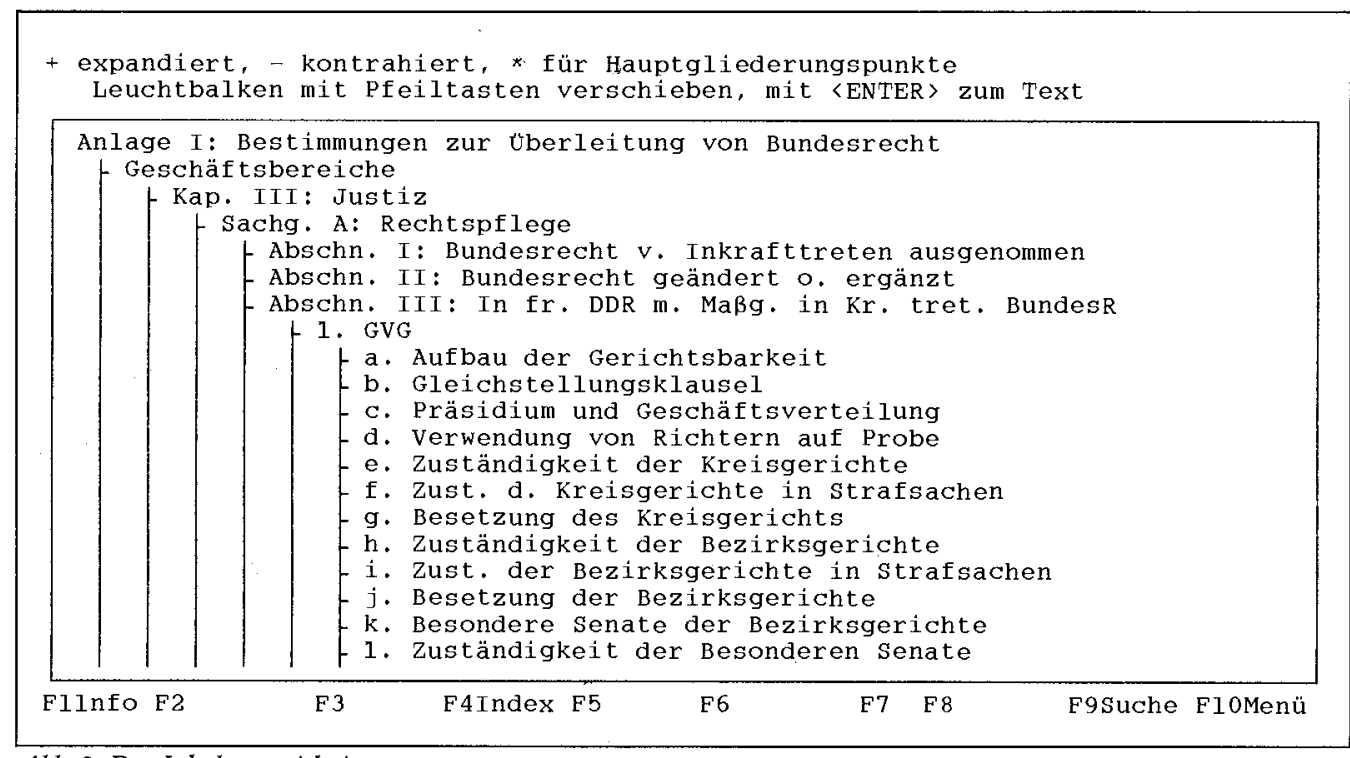

Abb. 2: Das Inhaltsverzeichnis.

Suchumgebungen der Fall ist. Im übrigen ist es nicht nur Gewohnheit, die ein gliederungsorientiertes Arbeiten rechtfertigt: Während die Volltextsuche strukturlos ist, zwingt das gliederungsorientierte Arbeiten zum Denken in Strukturen. Daß damit methodische Vorzüge verbunden sind, liegt auf der Hand.

Die Struktur des gestaffelten Zugriffs wurde nicht nur der konkreten Realisierung nach sondern auch abstrakt beschrieben, um deutlich zu machen, daß sie nicht allein für die Abbildung von Inhaltsverzeichnissen verwendet werden kann. Vielmehr kommt jegliche gestaffelte Liste als Anwendungsfall in Betracht. Beispielsweise könnte man alle im Einigungsvertrag genannten Fristen nach diesem Schema behandeln, indem man nach den Aspekten „Wer hat was bis wann zu tun?“ gliedert. Die entsprechenden Möglichkeiten sind also vielfältiger Art. Diese Vielfalt wird bei CDIS dadurch unterstützt, daß man aus mehreren Gliederungen auswählen kann. Zusätzlich erhöht wird die Flexibilität dadurch, daß die Gliederung nicht aufider CD-ROM enthalten sein muß, sondern stattdessen auch aufider Festplatte liegen kann. Aufi diese Art und Weise wird die nachträgliehe Erschließung des Informationsbestandes unabhängig von dem Vorgang der CDROM-Produktion unterstützt.

\section{Auf dem Weg zum eigenen Kommentar}

Eben war bereits von gewohnten Arbeitsweisen und der schrittweisen Verfeinerung des Informationsbestandes die Rede. In beiderlei Hinsicht schneidet das Buch gut ab: Juristen sind an die Arbeit damit gewöhnt und lieben es auch, Notizen anzubringen. So wächst das Buch mit dem eigenen Lernen und wird schließlich ein Unikat, ein persönliches Referenzmedium. Bei elektronischen Medien hat man bisher kaum Versuche unternommen, dieses „Mitwachsen“ im Laufe der Arbeit zu unterstützen. CD-ROM’s als vom Benutzer nicht beschreibbare Medien schließen sogar scheinbar eine derartige Möglichkeit von vornherein aus. Daß dem nicht so sein muß, zeigt CDIS. Hier kann der Benutzer einem Dokument eine Notiz hinzufügen, die zwar aufi der Festplatte gespeichert wird, aber immer wieder automatisch zur Verfügung steht, wenn die Suche erneut aufi das kommentierte Zieldokument stößt. Da derartige Notizen auch separat geliefert und durch bloßes Kopieren aufi die Festplatte über den Dateinamen mit Zieldokumenten aufi der CD-ROM kombinierbar sind, eröffnen sich ganz neue Möglichkeiten der Datenpflege und der Informationserweiterung.

\section{Auch der Einigungsvertrag: „hypertext by nature“}

Juristische Normtexte weisen meist komplizierte interne Verweisungen auf, was zu der These geführt hat, sie seien gewissermaßen naturgemäß Hypertext. Denn Hypertext bedeutet in einer allgemeinen Lesart nichts anderes, als daß verbundene Informationseinheiten „fest verdrahtet" werden. Das ist auch im Druck möglich und wurde in juri-

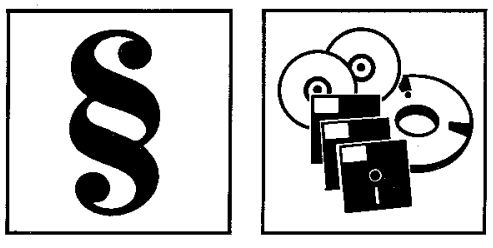

Mebrere Gliederungen sind möglich auch auf der. Festplatte und nacbträglich 
Realisierte HypertextVerknüpfungen stischen Texten stets so praktiziert. Ein „vgl. dazu oben S. 345“ läßt sich als HypertextLink verstehen. Nur ist das Verfolgen dieser so bezeichneten internen Querverbindung mit dem Aufwand des Blätterns verbunden, wobei es u.U. beim Weg über mehrere Stationen nicht einfach ist, die einzelnen Haltepunkte zu markieren. Von den damit gerade beim Einigungsvertrag verbundenen Schwierigkeiten war bereits eingangs mit Beispielen die Rede. Aufi der CD-ROM sind (ohne daß damit bereits das mögliche Optimum erreicht wäre) drei Arten von Hypertext-Verknüpfungen enthalten:

- Wenn aus den Anlagen, dem Gesetz, dem Protokoll oder dem Vertrag selbst auf einen Artikel des Vertrages verwiesen wird, ist der Text dieses Artikels „via Hypertext“ direkt erreichbar (vgl. Abb. 3)

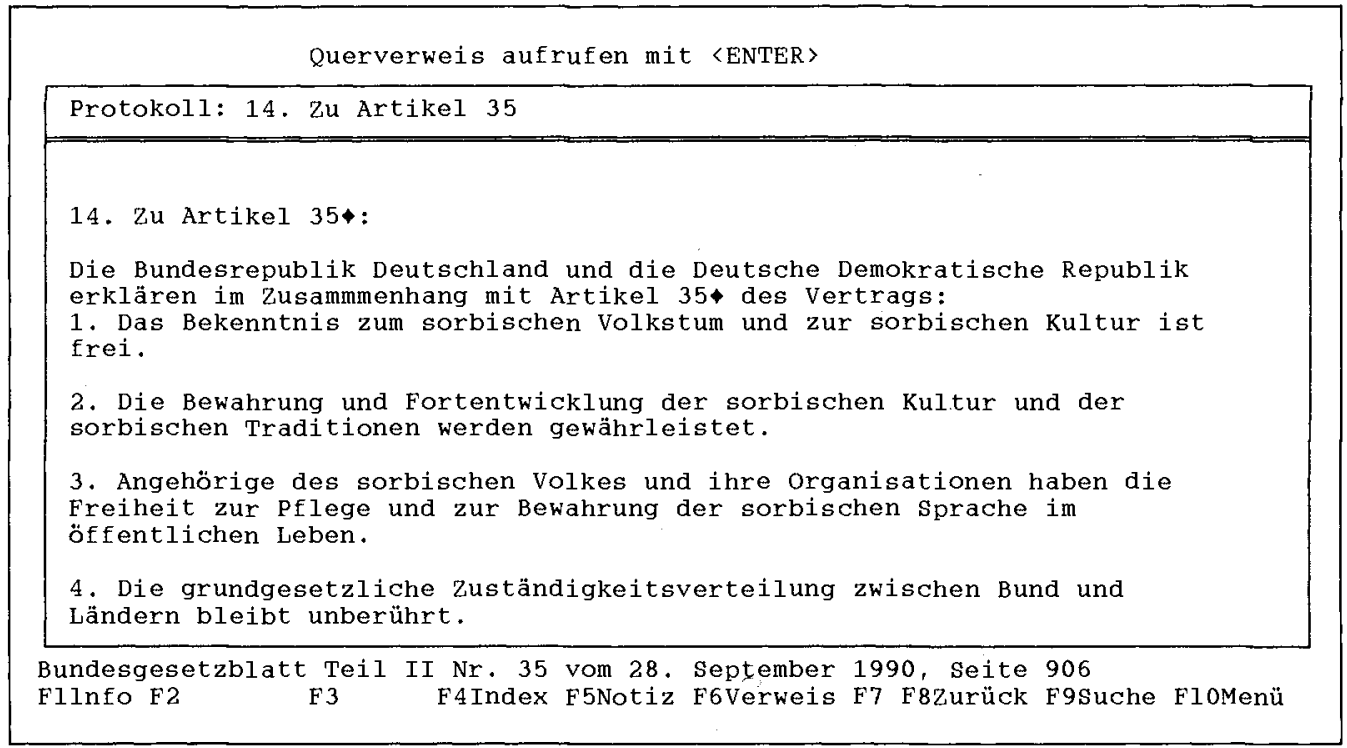

Abb. 3: Der Diamant - Symbol für Hypertext.

Als Hypertext aufgelöst sind die internen Querverweise des Komplexitätsgrades „Anlage $v$ Kapitel w Sachgebiet x Abschnitt y Nr. z“.

- Den Regelungen des Einigungsvertrages, die in der Vereinbarung zur Durchführung und Auslegung des Einigungsvertrages geändert worden sind, wurden diese Änderungen hypertext-verknüpft zugeschrieben.

Der zuletzt angesprochene Hypertext-Komplex demonstriert im übrigen auch, daß in CDIS Hypertext-Verknüpfungen kategorial qualifiziert werden können. Das ist deswegen wichtig, weil die pure Verknüpfung zweier Informationseinheiten Gefahr läuft, inhaltsarm zu sein. Sie besagt im Extremfall nur, daß etwas mit etwas anderem zusammenhängt. Der auf dieses schwache Hypertext-Konzept bezogenen Kritik ist dahingehend recht zu geben, daß eine genauere Typisierung der Art des Zusammenhangs möglich sein muß.

\section{Ausblick}

Die Arbeit mit dem Einigungsvertrag, dessen zentrale Bedeutung für unser Rechtssystem hier stillschweigend als anerkannt vorausgesetzt wurde, wird den juristischen Alltag der nächsten Jahre entscheidend mit prägen. Wenn die Herausforderung dieses Textes auch dazu genützt wird, unsere Arbeitsmittel und Suchmethoden nicht nur aufidie Probe zu stellen, sondern gleichzeitig weiterzuentwickeln, könnte für den juristischen Umgang mit komplexen Informationsmengen eine neue Dimension gewonnen werden. Eine Prognose darüber, ob die elektronischen Hilfsmittel in diesem Prozeß als unverzichtbar anerkannt werden, soll hier nicht gewagt werden. Da ohne sie eine strengsten Ansprüchen genügende Arbeit aber nicht möglich ist, steht doch zu hoffen, daß methoden- und qualitätsbewußte Juristen dieser Herausforderung nicht ausweichen werden. 